\title{
Mapeamento sistemático sobre sistemas de apoio ao professor na autoavaliação de suas aulas presenciais
}

\author{
Antônio Plínio Feitosa Bastos ${ }^{1}$, Elizabeth Sucupira Furtado \\ ${ }^{1}$ Programa de Pós-Graduação em Informática Aplicada (PPGIA) \\ Universidade de Fortaleza (Unifor) - Fortaleza, CE - Brasil \\ mr.pliniodgmail.br, elizabeteunifor.br
}

\begin{abstract}
This paper presents a systematic mapping aiming to identify systems that support the self-evaluation of face-to-face classes for professors, correlated to the lesson plan. In addition, among other factors, to verify what are their context of use and what are the forms of interaction they work. As result, a study was found using established criteria that portrays the lesson plan, but does not address self-evaluation. Thus, allowing the future design and development of a system with the sought characteristics.
\end{abstract}

Resumo. Este artigo apresenta um mapeamento sistemático com objetivo de identificar sistemas que deem suporte à autoavaliação de aulas presenciais para professores, correlacionado ao plano de aula. Além disso, dentre outros fatores, verificar quais são seus contextos de uso e quais são quais as formas de interação que eles trabalham. Como resultado, foi encontrado um estudo mediante critérios estabelecidos que retrate o plano de aula, mas que não aborda a autoavaliação. Assim, possibilitando a concepção e o desenvolvimento futuros de um sistema com as características procuradas.

\section{Introdução}

Professores universitários são incentivados a não se contentar em apenas transmitir conteúdos e conhecimentos científicos, mas em criar, ou seja, combinar docência com pesquisa. Além disso, são orientados a dar um sentido prático e profissionalizante à formação que oferece a seus alunos [Morgado 2015]. Durante o primeiro semestre de 2019, observou-se a dificuldade que professores universitários têm em se autoavaliar e acompanhar suas aulas de modo prático e fácil, principalmente mediante o curto intervalo de tempo entre as diversas aulas ao longo do dia. Verificar existência de sistemas que sanem essa dificuldade, contribuindo com a autoavaliação de suas aulas em tempo hábil e cruzando com objetivos educacionais sem se apresentar como uma tarefa ineficiente e/ou árdua, tornou-se a problemática deste mapeamento sistemático.

O mapeamento sistemático é uma revisão ampla de estudos primários existentes em um tópico específico de pesquisa, objetivando caracterizar uma área de pesquisa, a partir da quantificação, categorização e análise dos estudos publicados, utilizando uma metodologia rigorosa e confiável. O processo de um mapeamento sistemático envolve três fases: planejamento, condução e publicação dos resultados. Cada uma das fases tem atividades específicas conduzidas de forma iterativas. Para a primeira fase, têm-se as atividades: definir objetivos; definir protocolo, e; avaliar protocolo. Já na segunda fase, têm-se: identificar estudos primários; selecionar estudos primários; extrair os dados, e; sintetizar os dados. Por fim, a última fase envolve: descrever e divulgar os resultados, e; 
VIII Congresso Brasileiro de Informática na Educação (CBIE 2019)

Anais do XXV Workshop de Informática na Escola (WIE 2019)

avaliar os resultados relatados [Cooper 2016; Kitchenham and Charters 2007; Arksey and O'Malley 2005].

As seções seguintes apresentam o detalhamento do mapeamento sistemático realizado neste estudo sobre sistemas de apoio ao professor na autoavaliação de suas aulas presenciais, realizado entre janeiro e maio de 2019 .

\section{Planejamento do Mapeamento Sistemático}

O objetivo do mapeamento sistemático foi prover estudo/pesquisa que identifique requisitos e aplicações de sistemas tecnológicos que deem suporte ao plano de aula e à autoavaliação de aulas ministradas. De modo particular, identificar quais soluções são voltadas a professores, analisando se o que eles planejaram foi efetivamente realizado em sala de aula. É importante que a solução seja fácil de usar e com a percepção de ganhos, para não requerer mais outro esforço do professor.

O protocolo é o elemento fundamental para a execução do mapeamento sistemático. Relata as questões de pesquisa, a estratégia utilizada para conduzir o mapeamento sistemático, os critérios para a seleção de estudos e como os dados obtidos serão extraídos dos estudos e como serão sintetizados [Falbo 2015]. Assim, para auxiliar a elaboração das questões de pesquisa, foi utilizada a técnica PICOC, do inglês: Population, Intervention, Comparison, Outcome e Context [Petticrew and Roberts 2006]. Assim, foram estabelecidos: P - professores; I - ferramenta de autoavaliação da aula ministrada; $\mathrm{C}$ - sem grupo de controle, por ter caráter exploratório; $\mathrm{O}$ - sistemas de autoavaliação da aula, requisitos e/ou validação desses sistemas, e; C - artigos com sistema, requisitos e/ou validação. Desse modo, como resultado foram elaboradas cinco questões de pesquisa: Q1 - 'Quais sistemas ou ferramentas tecnológicos utilizados para plano de aula e para autoavaliação de aula ministrada e em que contexto de uso estão inseridos?'; Q2 - 'Quais são os requisitos definidos para as soluções?'; Q3 - 'Quais são as formas de validação das soluções?'; Q4 - 'Quais critérios de autoavaliação são utilizados nas soluções?', e; Q5 - 'Quais formas de interação são dadas pelas soluções?'.

Ao idealizar a estratégia do protocolo, foram definidos métodos de busca, fontes de pesquisa, string de busca a ser usada, critérios de seleção e etapas do processo de seleção a serem realizadas. Optou-se por um método de busca automática em cinco bases digitais: Scielo, IEEE Xplore, Renote, Rbie e Sbie; em português ou em inglês, e; com trabalhos publicados a partir de 2000. A string inicial de busca foi definida como: (class OR classroom) AND (evaluation OR self-evaluation OR valuation) AND (system OR tool OR software OR framework). Para os critérios de inclusão, foram escolhidos trabalhos que: propõem ferramenta ou sistema ou método ou metodologia ou processo tecnológico voltado para área de educação, em especial que envolva autoavaliação do professor e/ou acompanhamento do planejamento didático ou plano de aula; apresentam seus requisitos; realizam sua validação, e; descrevem critérios para autoavaliação.

De modo análogo aos critérios de inclusão, os critérios de exclusão definidos foram: estar fora dos idiomas propostos; estar indisponível ou ser pago; estar duplicado; não possuir resumo/abstract; não estar completo; estar fora do escopo, como ser voltado para avaliação do aluno ou para ensino à distância; não possuir autor; estar fora das áreas de computação ou de educação, e; não ser artigo de conferência ou periódico. A busca e a seleção foram feitas em duas etapas: leitura de resumo/abstract, e; caso passasse nos critérios, seria feita uma leitura integral do trabalho. 
VIII Congresso Brasileiro de Informática na Educação (CBIE 2019)

Anais do XXV Workshop de Informática na Escola (WIE 2019)

O protocolo completo foi avaliado por um grupo de sete pesquisadores da área de Interação Humano-Computador do Programa de Pós-Graduação em Informática Aplicada (Universidade de Fortaleza), onde foram sugeridas modificações de melhoria na string inicial de busca, para garantir uma maior abrangência. As modificações foram validadas pelos autores e aplicadas, obtendo a string: (class OR classroom OR teacher OR professor OR lesson plan) AND (evaluation OR self-evaluation OR verification) AND (system OR tool OR software OR framework OR method OR methodology OR process).

\section{Condução do Mapeamento Sistemático}

Utilizando a string de busca modificada e os critérios de inclusão propostos na estratégia do protocolo, foram encontrados um total de 15.365 estudos nas bases digitais, sendo: Scielo (663), IEEE Xplore (14.441), Renote (3), Rbie (28) e Sbie (230). Após aplicar os critérios de exclusão definidos no protocolo, o total passou para 265 estudos: Scielo (137), IEEE Xplore (78), Renote (1), Rbie (28) e Sbie (21), mantendo a contagem na base mais à esquerda o trabalho que estivesse duplicado nas bases seguintes. Finalizando a primeira etapa (leitura dos resumos/abstracts) e a segunda etapa (leitura integral dos estudos), foram selecionados um total de 9 estudos, distribuídos nas bases: Scielo (6), IEEE Xplore (1), Renote (0), Rbie (2) e Sbie (0).

Foi decidido avaliar a qualidade dos trabalhos selecionados. Recomenda-se usar critérios de qualidade que se possam ser aplicados em diferentes tipos de trabalhos [Wolin et al. 2013]. Desse modo, foram criados sete critérios de qualidade: apresentar software, ferramenta ou sistema tecnológico, voltado para área de educação (C1); ser direcionado à autoavaliação do professor (C2); apresentar requisitos da solução (C3); apresentar validação da solução (C4); ser ambientado no ensino superior (C5); trazer formas de interação da solução (C6), e; mostrar critérios de autoavaliação (C7).

Os estudos selecionados receberam um código: Barbosa e de Oliveira (2004) E1; Gomes et al. (2013) - E2; Alves et al. (2018) - E3; Cassettari (2017) - E4; Rowe, Bastos e Pinho (2013) - E5; Grohmann e Ramos (2012) - E6; Bertolin e Marchi (2010) E7; Nunes e Helfer (2009) - E8, e; Xu e Wang (2018) - E9. E, para cada critério de qualidade, os estudos pontuaram em: C1 - E1; C2 - E3, E4, E5, E6, E7, E8, E9; C3 - E1, E2; C4 - E2; C5 - E5, E6, E7, E8, E9; C6 - Ø, e; C7 - E3, E6, E7, E8. Ou seja, nenhum trabalho encontrado com mais da metade dos critérios de qualidade estabelecidos.

\section{Publicação dos Resultados}

O mapeamento sistemático, obtido após seu planejamento, sua condução e a avaliação de seus resultados, foi descrito em forma de artigo resumido para ser submetido ao WIE 2019 - Workshop de Informática na Escola -, evento que faz parte do CBIE 2019 Congresso Brasileiro de Informática da Educação.

Dentre os nove estudos selecionados apenas um deles se trata de um software/ferramenta voltado para área de educação (E1) - os demais estudos trazem resultados de pesquisas ou descrições pedagógicas sobre avaliação do professor -, abordando a questão do plano de aula, mas não trazendo a autoavaliação do professor. Grande parte dos estudos, 78\% deles, trazem uma abordagem de autoavaliação (E3, E4, E5, E6, E7, E8, E9), mas de um modo genérico ou como parte de uma avaliação institucional. Isto é, trata-se da avaliação feita pelo professor de um semestre ou de um 
ano inteiro sobre estrutura institucional. Nenhum desses estudos traz a autoavaliação de uma aula que um professor tenha acabado de ministrar ou o plano de aula.

Dentre outros fatores avaliados de modo geral, alguns deles são: didática ou habilidades acadêmicas (E3, E4, E6, E7, E8, E9), tempo de serviço na área (E3), estrutura institucional (E4, E6), conhecimentos ou carreira (E5, E6, E8), cumprimento dos objetivos pedagógicos (E3, E7), relacionamento com alunos (E3, E6, E8, E9), avaliações pedagógicas (E3, E6, E9) e obrigações (E3, E4, E5, E6), como pontualidade. Esses fatores fazem parte de um plano de aula. A didática geral preza que para planejar uma aula é necessário pensar no que o aluno deve aprender, isto é: pensar nos objetivos de aprendizagem, nos conteúdos a ser ensinado, nos meios pelos quais serão desenvolvidos tais conteúdos e na avaliação da aula [Schewtschik 2017].

Parte dos estudos, 56\%, são voltados para o âmbito do ensino superior (E5, E6, E7, E8, E9), o que indica uma frente de pesquisa voltada ao professor universitário, buscando acompanhar seu desempenho. E, baseando-se nas questões de pesquisa, não foram encontrados sistemas que abordem autoavaliação (Q1). Desse modo, como não foram encontrados estudos que atendessem a Q1, consequentemente não atenderam à Q2, Q3, Q4 e Q5. Apesar de não ser voltado para autoavaliação, E1 traz requisitos de cadastro, acesso e controle acadêmico (planejamento didático) em um software e E2 traz requisitos de uma ferramenta tecnológica, o hardware (Q2). Adicionalmente, após leituras completas, não foram identificados estudos que descrevessem ou focassem em formas de interação humano-computador (Q5).

\section{Conclusões}

Após planejamento, condução e avaliação de um mapeamento sistemático sobre sistemas de apoio ao professor na autoavaliação de suas aulas presenciais, foi identificado apenas um estudo que aborde sistema voltado para o plano de aula, mas que não inclui a autoavaliação de aulas presenciais ministradas por professores. Assim, justifica-se o estudo, a concepção, o desenvolvimento e a análise de interação futuros de tal sistema. Para o sistema, vislumbra-se: correlacionar aos aspectos do plano de aula; utilizar uma forma de interação fácil e prática para o dia-a-dia do professor; ser voltado para o ambiente universitário, e; fazer o acompanhamento do planejamento didático.

\section{Agradecimentos}

Ao Programa de Pós-Graduação em Informática Aplicada (PPGIA) da Universidade de Fortaleza (Unifor) pelo espaço e infraestrutura para a execução da pesquisa e à Fundação Cearense de Apoio ao Desenvolvimento Científico e Tecnológico (Funcap) pelo suporte no financiamento da pesquisa.

\section{Referências}

Alves, M. P. et al. (2018) "Perceção dos professores sobre a avaliação do desempenho docente", Revista Portuguesa de Educação, vol. 31, 1, pages 61-78. DOI: https://doi.org/0.21814/rpe.14082.

Arksey, H. and O'Malley L. (2005) "Scoping studies: towards a methodological framework", International Journal of Social Research Methodology, vol. 8, 1, pages 19-32. DOI: https://doi.org/10.1080/1364557032000119616. 
VIII Congresso Brasileiro de Informática na Educação (CBIE 2019)

Anais do XXV Workshop de Informática na Escola (WIE 2019)

Barbosa, F. K and de Oliveira, C. A. S. (2004) "SACAD - Sistema de avaliação, controle e apoio didático", UNILUS Ensino e Pesquisa, vol. 1, 1, pages 51-60. http://revista.unilus.edu.br/index.php/ruep/article/view/1.

Bertolin, J. C. G. and de Marchi, A. C. B. (2010) "Instrumentos para avaliar disciplinas da modalidade semipresencial: uma proposta baseada em sistemas de indicadores", Avaliação da Educação Superior, vol. 15, 3, pages 131-146. DOI: http://dx.doi.org/10.1590/S1414-40772010000300007.

Cassettari, N. (2017) "Avaliação de professores: relato da construção de uma proposta", Cadernos de Pequisa, vol. 47, 165, pages 1070-1090. DOI: 10.1590/198053143944.

Cooper, I. D. (2016) "What is a mapping study?", J Med Lib Assoc, vol. 104, 1, pages 76-78. DOI: 10.3163/1536-5050.104.1.013.

Falbo, R. A. (2015) "Mapeamento Sistemático", https:/www.inf.ufes.br/ falbo/files/ MP/TP/Sobre_MS.pdf, January.

Gomes, A. S. et al. (2013) "Design of a Self-standing Multimedia Enriched Projector to enhance teaching experience in classroom in Brazilian public schools", Revista Brasileira de Informática na Educação, vol. 21, 1, pages 23-36. DOI: 10.5753/RBIE.2013.21.01.23.

Grohmann, M. Z. and Ramos, M. S. (2012) "Competências docentes como antecedentes da avaliação de desempenho do professor: percepção de mestrandos de administração", Avaliação do Ensino Superior, vol. 17, 1, pages 65-86. DOI: http://dx.doi.org/10.1590/S1414-40772012000100004.

Kitchenham, B. and Chaters, S. (2007) "Guidelines for performing Systematic Literature Reviews in Software Engineering", Keele University and University of Durham. http://citeseerx.ist.psu.edu/viewdoc/summary?doi=10.1.1.117.471.

Morgado, J. C. (2015) "La enseñanza universitária - El escenario y sus protagonistas", Educar em Revista, vol. 57, pages 293-301. DOI: 10.1590/0104-4060.42072.

Nunes, A. K. and Helfer, C. L. L. (2009) "Diagnóstico do desempenho na docência da graduação da UNISC", Avaliação da Educação Superior, vol. 14, 1, pages 169-183. DOI: http://dx.doi.org/10.1590/S1414-40772009000100009.

Petticrew, M. and Roberts, H. (2006), Systematic Reviews in the Social Sciences, Blackwell Publishing Ltd., Oxford. DOI: 10.1002/9780470754887.

Rowe, D. E. O; Bastos, A. V. B. and Pinho, A. P. M. (2013) "Múltiplos comprometimentos com o trabalho e suas influências no desempenho: um estudo entre professores do ensino superior no brasil", Organizações \& Sociedade, vol. 20, 66, pages 501-522. DOI: http://dx.doi.org/10.1590/S1984-92302013000300008.

Schewtschik, A. (2017) "O planejamento de aula: um instrumento de garantia de aprendizagem”, Educere - XIII Congresso Nacional de Educação, p. 10661-10677.

Wohlin, C. et al. (2013) "On the reliability of mapping studies in software engineering", Journal of Systems and Software, vol. 86, 10, pages 2594-2610. DOI: 10.1016/j.jss.2013.04.076.

$\mathrm{Xu} \mathrm{X}$. and Wang Y. (2018) "Teaching performance evaluation in smart campus", IEEE Access, vol. 6, pages 77754-77766. DOI: 10.1109/ACCESS.2018.2884022. 